\title{
LIMB-GIRDLE MUSCULAR DYSTROPHY TYPE 2B AS A DIFFERENTIAL DIAGNOSIS OF REFRACTORY POLYMYOSITIS: A CASE REPORT
}

Jucier Gonçalves Júnior ${ }^{1, \star}$, Fernando Henrique Carlos de Souza ${ }^{1}$, Renata Miossi ${ }^{1}$, Maria Eugênia Teixeira Bicalho ${ }^{1}$, Samuel Katsuyuki Shinjo ${ }^{1}$

1.Universidade de São Paulo, São Paulo (SP), Brazil.

*Corresponding author: juciergjunior@hotmail.com

\section{BACKGROUND}

Polymyositis (PM) is a rare systemic autoimmune myopathy with related diagnoses that need to be constantly considered, especially in refractory cases. The present study represents a case of refractory PM with a confirmed diagnosis of limb-girdle muscular dystrophy type 2B (LGMD2B).

\section{CASE REPORT}

A black female patient, 51 years old, presented with a history of objective muscle weakness, predominantly proximal to the four limbs over the past two years. The patient exhibited electroneuromyography with a diffuse predominantly proximal myopathic pattern and persistent elevation of creatine phosphokinase (maximum $5034 \mathrm{U} / \mathrm{L}$ ) and aldolase (maximum $15.1 \mathrm{U} / \mathrm{L}$ ) serum levels. The patient had also had two muscle biopsies (brachial biceps and quadriceps femoris) with nonspecific myositis pattern. With the possible diagnosis of PM, she received glucocorticoid (maximum $1 \mathrm{mg} / \mathrm{kg} /$ day) in addition to methylprednisolone pulse therapy (1000 mg/day for 3 days) on more than one occasions, several immunosuppressive drugs (methotrexate, azathioprine cyclosporine, cyclophosphamide - as monotherapy or in combination), and biological drugs (rituximab and abatacept). Due to the refractoriness of the disease, we proceeded with a third biopsy at a 13-year follow-up, in which a myopathic and dystrophy patterns were evidenced, in addition to deficiency of dysferlin; therefore, the new diagnosis was LGMD2B. The current medications (methotrexate and cyclosporine) were suspended and the dose of glucocorticoid decreased. The patient was referred to the neuromuscular unit of our service for following-up and clinical management.

\section{CONCLUSION}

Dysferlinopathies such as LGMD2B are rare and differential diagnose with PM are essential for early adequate psychological and physiotherapy support and avoiding unnecessary therapeutic interventions.

\section{KEYWORDS}

Case report, Limb-girdle muscular dystrophy type 2B, Polymyositis, Rituximab. 